\title{
Quantitative Nanomechanical Property Mapping and in situ SPM Imaging of Polyetherimide Nanocomposites
}

\author{
Farooq Syed ${ }^{1}$, Shaik Zainuddin ${ }^{1 *}$, Isaiah Wilson ${ }^{1}$, Mohamed Elafandi ${ }^{1}$ and Shaik Jeelani ${ }^{1}$ \\ 1. Department of Materials Science and Engineering, Tuskegee University, Tuskegee, AL, USA. \\ * Corresponding author: szainuddin@tuskegee.edu
}

Determination of mechanical properties of nanocomposites through conventional macroscale test methods is always challenging and questionable. Nanoparticles interact with polymer molecules at a micron to nanoscale, and hence to precisely determine the properties of these composites, micron level test methods such as nanoindentation need to be utilized. In this work, we have used the nanoindentation techniques combined with a recently developed novel Accelerated Property Mapping (XPM) technique to study the statistical quantification of mechanical properties such as hardness and modulus, and the variance of these properties across the phases of Polyetherimide (PEI) nanocomposites reinforced with Zinc titanate $\left(\mathrm{ZnTiO}_{3}\right)$ nanoparticles. Polyetherimide is a high-performance thermoplastic polymer with excellent mechanical and tribological properties even at elevated temperature due to its high glass transition temperature [3]

Polyetherimide (PEI) pellets with a melt index of $18 \mathrm{~g} / 10 \mathrm{~min}\left(337^{\circ} \mathrm{C}\right)$ and density of $1.27 \mathrm{~g} / \mathrm{mL}$ at $25^{\circ} \mathrm{C}$ was purchased from Sigma-Aldrich. Zinc titanate $\left(\mathrm{ZnTiO}_{3}\right)$ nanopowder with a molecular weight of $161.26 \mathrm{~g} / \mathrm{mol}$ and a $<100 \mathrm{~nm}$ particle size (BET), 99\% trace metals basis and along with $\mathrm{N}, \mathrm{N}-$ Dimethylacetamide was also purchased from Sigma-Aldrich.

At first, 15 gm of PEI pellets were mixed with $100 \mathrm{ml}$ of $\mathrm{N}, \mathrm{N}$-Dimethylacetamide $\left(1000 \mathrm{rpm}\right.$ at $\left.150{ }^{\circ} \mathrm{C}\right)$ for three hours until all the pellets were completely dissolved. The solution was then poured in Aluminum pans and placed on the hot plate for curing at $140{ }^{\circ} \mathrm{C}$ for 72 hours. Finally, the samples were removed and carefully polished using alumina suspension and mounted on a circular metallic puck of 13 $\mathrm{mm}$ in diameter using a cyanoacrylate glue.

Quantitative mapping of hardness and modulus were determined by Accelerated Property Mapping (XPM) technique using Hysitron TI-980 Tribonanoindenter with a diamond Berkovich tip. XPM is a recently developed technique through which the distribution of mechanical properties along the phases of material at nanolevel can be determined and mapped [1-2]. To acquire the XPM maps, a constant force of $200 \mu \mathrm{N}$ with an indent spacing of $1000 \mathrm{~nm}$ was used over a $20 \times 20 \mu \mathrm{m}$ area. Several tests were carried out with 100 indents in each test for quantitative hardness and modulus maps. The EDS spectrum was acquired using Field Emission Scanning Electron Microscope (JSM-7200F) equipped with Energy Dispersive X-Ray Analyzer, EX-37001.

The hardness maps of PEI neat and 5 wt. \% samples showed an average value of $0.48 \mathrm{GPa}$ (Fig. 1c) and $0.55 \mathrm{GPa}$ (Fig. 2c), respectively. A significant increase of $14.59 \%$ was found in 5 wt. \% PEI samples compared to its neat counterpart. Similarly, the modulus map of 5 wt. \% PEI/ZnTiO 3 showed 6. $38 \mathrm{GPa}$ in comparison to $5.64 \mathrm{GPa}$ observed in neat PEI samples with an improvement of $13.12 \%$ in modulus. The EDS spectrum of PEI- $\mathrm{ZnTiO}_{3}$ nanocomposites shows the elemental composition of $\mathrm{PEI} / \mathrm{ZnTiO}_{3}$ and the presence of $\mathrm{ZnTiO}_{3}$ nanoparticles in the PEI polymer (Fig. 1b). Similar results were also reported by Yildiz, $\mathrm{K}$ et al., [4,5]. 
References:

[1] ED Hintsala, U Hangen and DD Stauffer 70 (2018), p. 1.

[2] D Li, Fastmap High Speed Nanoindentation Mapping (2015), p. 1.

[3] S Kumar et al., Materials Science and Engineering: B 141(1-2), 61-70 (2007), p. 1.

[4] K Yildiz et al., Materials Science-Poland 25 (2007), p.1.

[5] The authors acknowledge funding from the NSF-MRI \# 1725513.
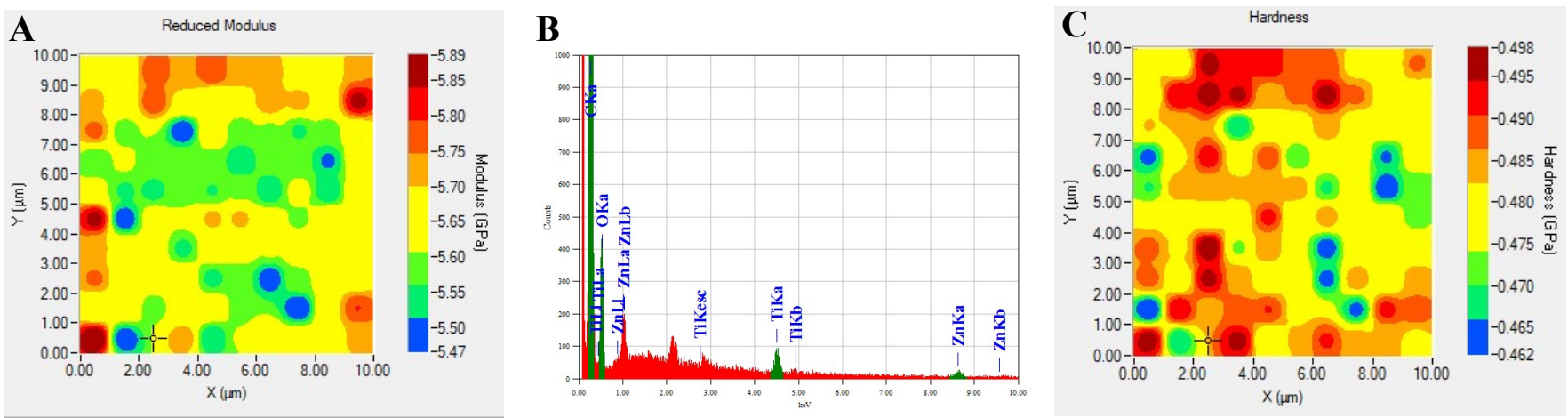

Figure 1. A) An image showing the modulus for a neat sample. B) EDS Spectrum of PEI- $\mathrm{ZnTiO}_{3} \mathrm{C}$ ) An image showing the harness for a neat sample
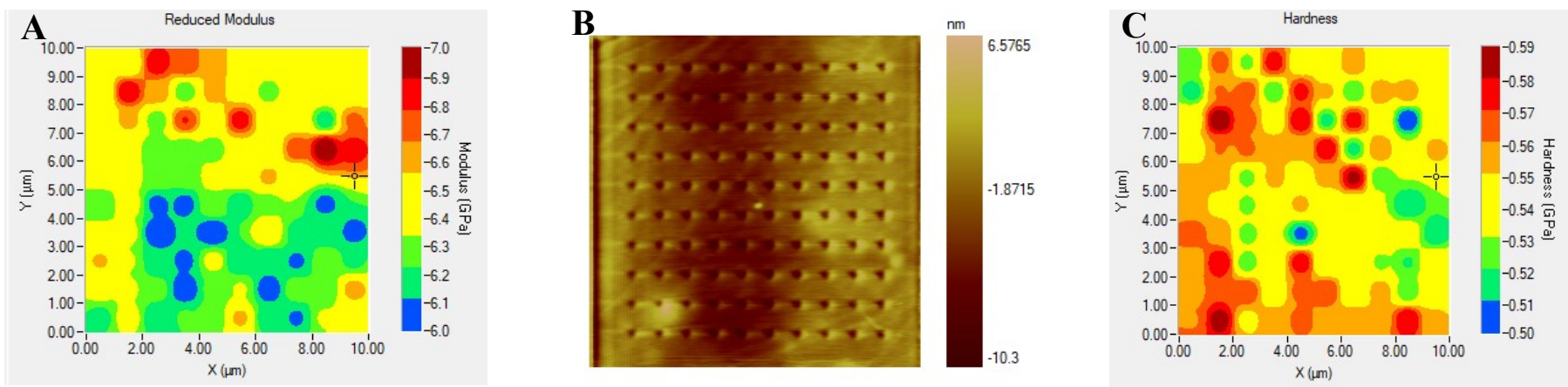

Figure 2. A) An image showing the modulus for a 5 wt. \% sample. B) SPM image C) An image showing the harness for a $5 \%$ sample 